\title{
The Coronal Structure of the Sun-Like Exoplanet-Host GJ 3021
}

\author{
J. D. Alvarado-Gómez ${ }^{1}$, O. Cohen ${ }^{2}$, G. A. J. Hussain ${ }^{1}$, J. Grunhut ${ }^{1}$, \\ C. Garraffo ${ }^{2}$ and J. Drake ${ }^{2}$ \\ ${ }^{1}$ ESO, Karl-Schwarschild-Str. 2, D-85748, Garching bei München, Germany \\ email: jalvarad@eso.org \\ ${ }^{2}$ Harvard-Smithsonian Center for Astrophysics, 60 Garden Street, Cambridge, MA 02138, USA
}

\begin{abstract}
We present the results of a numerical simulation of the corona and wind structure of the Sun-like exoplanet-host GJ 3021 using a global magnetohydrodynamic (MHD) model. The simulation is driven by the radial component of the surface magnetic field recovered with the Zeeman Doppler Imaging (ZDI) technique. We consider two different ZDI input maps, which have similar large-scale structures but different spatial resolutions and field strengths. These maps arise from different but comparable models used to fit the observed circularly polarised spectra of the star. Our simulations show that the structure of the inner corona is consistent among the considered cases. Larger discrepancies are found in the wind structure, in particular in the radial wind speed and the Alfvén surface topology. These elements can have a significant impact on the mass loss and angular momentum loss predicted for this system and in other studies based on this numerical data-driven approach.
\end{abstract}

Keywords. Stars: coronae, stars: magnetic fields, stars: mass loss, stars: winds, outflows

\section{Introduction}

Solar and stellar winds are of central importance to explain the evolution of cool star systems in the early main sequence. $\mathrm{G}$ to $\mathrm{K}$ type stars tend to rotate rapidly on the Zero Age Main Sequence; braking torques exerted by winds (or outflows) cause them to spin down, losing most of their angular momentum within the first 500 Myr on the main sequence. Strong winds from the young Sun have been used to explain both the stripping of the Martian atmosphere (Terada et al. 2009), and the "Faint Sun paradox". This paradox states that geological records indicate that water existed in liquid form very early in the history of Earth despite the young Sun having only $70 \%$ of its current luminosity. Shaviv (2003) suggested that this discrepancy can be explained by a significantly stronger solar wind. Since the winds and outflows are magnetically driven phenomena in Sun-like stars, a correlation with the magnetic field is expected. However, while the young Sun was more magnetically active it does not necessarily follow that it would have hosted stronger winds (Wood et al. 2014).

The large-scale surface magnetic field topology in stars different than the Sun can be retrieved to some extent using the technique of Zeeman Doppler Imaging (ZDI) (Hussain et al. 2009 and references therein). Numerous studies have shown how ZDI maps can be used to model the circumstellar environment, including coronal X-ray emission (e.g. Hussain et al. 2007), winds and outflows (e.g. Cohen et al. 2010, Vidotto et al. 2011) and the evolution of planetary systems via star-planet interactions (e.g. Cohen et al. 2014). However, as with similar tomographic inversion techniques, the robustness and spatial resolution of the ZDI maps depend strongly on the phase-coverage of the observations and the rotational velocity, $v \sin (i)$, of the star. Moreover, stellar inclination and flux 


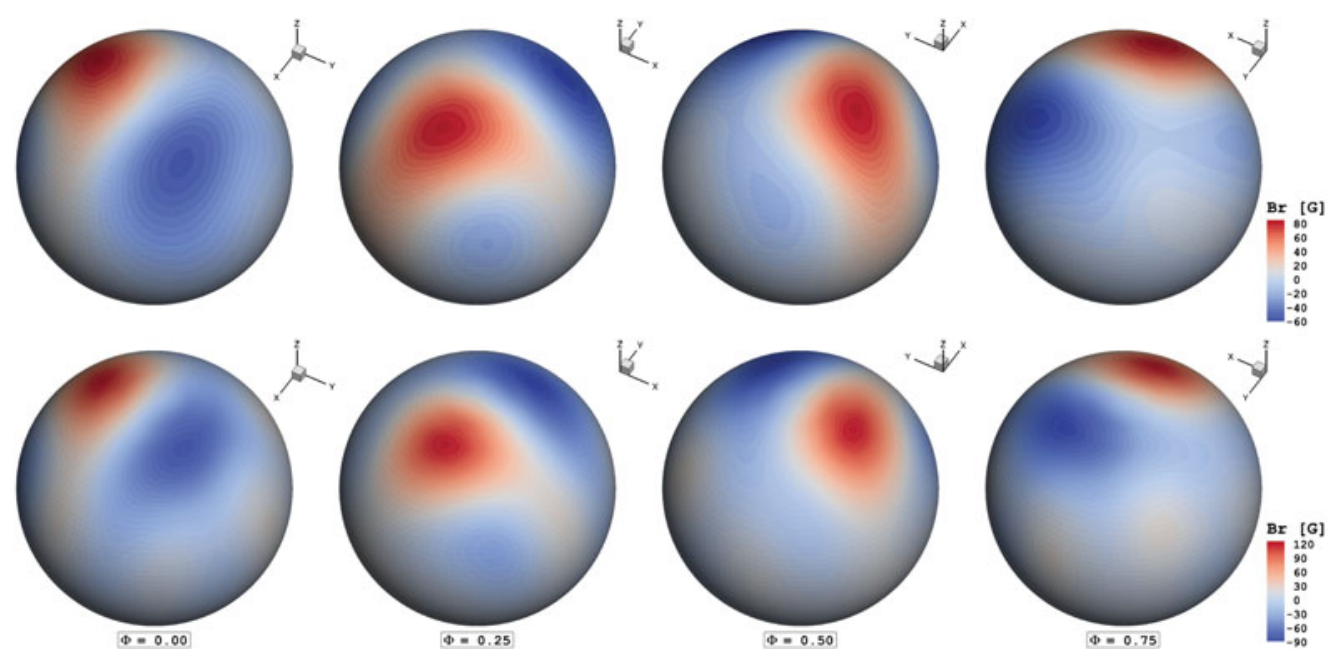

Figure 1. Recovered ZDI maps of the surface magnetic field of GJ 3021. The colour scale indicates the polarity and the field magnitude in Gauss $(G)$. Each row (Case A: top, Case B: bottom) corresponds to a different ZDI map in terms of the magnetic field strength, topology and spatial resolution. Four rotational phases $(\Phi)$ are displayed in each case. See the on-line edition of this book for a color version of this figure.

cancellation effects could lead to a considerable fraction of missing magnetic field information in the ZDI map (see Hussain \& Alecian 2014). These factors are of course different in each system and, therefore, the comparisons between ZDI studies (and models derived from them) are not straightforward. This could be particularly important when these type of observations and models are used to study relations between stellar magnetism and other astrophysical quantities (Vidotto et al. 2014).

In this proceeding, we present the results of a numerical simulation of the corona and wind structure of the Sun-like exoplanet-host GJ 3021, using a three-dimensional magnetohydrodynamic (MHD) model. The simulation is driven by the radial component of the large-scale surface magnetic field recovered with ZDI. For our simulation we use a global MHD model developed for the solar corona, which provides a self-consistent stellar wind solution, having the magnetic field distribution as a boundary condition. Two ZDI maps having similar large-scale structures but different spatial resolution and field strengths are used in the model. These differences arise from the observational procedure itself, allowing us to estimate the impact of these factors on the model in connection with the capabilities of ZDI. We present the input ZDI maps of GJ 3021 and the adopted stellar properties used in the simulation in Section 2. Section 3 contains a description of the numerical model and a discussion of the results is presented in Section 4. Conclusions and future prospects are presented in Section 5 .

\section{The Sun-Like Exoplanet-Host GJ 3021}

GJ 3021 (HD 1237) is a yellow-orange dwarf star of spectral type G8V, which has almost the same mass as the Sun, $86 \%$ of its diameter and $64 \%$ of its bolometric luminosity (Torres et al. 2006, Ghezzi et al. 2010). It is a young ( $<1$ Gyr), chromospherically active and confirmed exoplanet-host Sun-like star (Naef et al. 2001), making it a very interesting target for understanding its coronal structure and circumstellar environment.

In a previous work, we have recovered the surface magnetic field distribution of this star using ZDI from a time-series of high-resolution spectropolarimetric observations from HARPSpol. The reader is referred to Alvarado-Gómez et al. (2015) for further details. 
Figure 1 shows the input ZDI maps of GJ 3021 used in the simulation. Each row shows views of one map at four different rotational phases $(\Phi)$. The $\mathrm{z}$-axis is aligned with the rotation axis of the star. The colour scale indicates the polarity and the magnitude in Gauss $(\mathrm{G})$ of the radial component of the magnetic field. Each map has a different spatial resolution; $10.6^{\circ} / \mathrm{px}$ (Case A: top) and $6.0^{\circ} / \mathrm{px}$ (Case B: bottom). These differences arise from the line profile modelling, used in the ZDI magnetic field reconstruction process, and result in different magnetic field strengths and topologies. The 3D visualisations presented in Fig. 1, consider an inclination angle of the star $\left(\sim 42^{\circ}\right)$. For the simulations presented here, no assumptions are made regarding the magnetic field structure in the hidden fraction of the stellar surface. We will address this issue in a follow-up paper.

In addition to the surface magnetic field distribution, the model requires information about the coronal base density, $\rho_{0}$, and temperature, $T_{0}$, as well as the stellar mass, $M_{*}$, radius, $R_{*}$ and rotation period, $P_{\text {rot }}$, of the star. Table 1 contains a summary of the adopted stellar properties with their corresponding references.

Table 1. Adopted stellar properties for GJ 3021.

\begin{tabular}{|c|c|c|}
\hline Parameter & Value & References \\
\hline$n_{0}$ & $9.4 \times 10^{17} \mathrm{~m}^{-3}$ & Jordan et al. $(2001)^{1}$ \\
$T_{0}$ & $5.0 \times 10^{4} \mathrm{~K}$ & Sokolov et al. $(2013)^{2}$ \\
$M_{*}$ & $1.0 \mathrm{M}_{\odot}$ & Ghezzi et al. $(2010)$ \\
$R_{*}$ & $0.86 \mathrm{R}_{\odot}$ & Ghezzi et al. $(2010)$ \\
$P_{\text {rot }}$ & 7.0 days & Alvarado-Gómez et al. $(2015)$
\end{tabular}

Notes:

${ }^{1}$ Scaled value from the chromospheric diagnostics of the $\mathrm{K} 2 \mathrm{~V}$ dwarf $\epsilon$ Eridani. The listed value has been enhanced by a factor of 10 to avoid chromospheric evaporation (see van der Holst et al. 2014).

${ }^{2}$ Standard parameter assumed in the implemented coronal heating scheme (see Sect. 3 ).

\section{Numerical Simulation}

For our simulations we use the generic three-dimensional MHD code BATS-R-US (Powell et al. 1999) as part of the Space Weather Modelling Framework (SWMF, Tóth et al. 2012). The stellar wind solution is calculated using the up-to-date Stellar Corona (SC) module of the SWMF, where the initial condition for the entire simulation domain is given by a three-dimensional potential field extrapolation above the stellar surface. This initial extrapolation is performed based on the photospheric radial magnetic field of the star (Sect. 2). The simulation then evolves self-consistently calculating the coronal heating and the stellar wind acceleration due to Alfvén wave turbulence dissipation, taking into account electron heat conduction and radiative cooling effects. Further details can be found in Sokolov et al. (2013) and van der Holst et al. (2014).

The simulations of the corona and wind structure of GJ 3021 cover up to $30 R_{*}$, starting from the surface. We consider a non-uniform spherical grid, which is dynamically refined at the locations of magnetic field inversion. A maximum resolution of $1.2 \times 10^{-2} R_{*}$ is achieved. The simulation evolves until a steady-state solution is obtained. From this solution, all the coronal properties and stellar wind parameters, such as number density, $n$, plasma temperature, $T$, velocity, $\mathbf{u}$ and magnetic field, $\mathbf{B}$, are extracted.

\section{Results}

The 3D steady-state MHD solutions of the corona of GJ 3021 are presented in Figs. 2 and 3. Cases A and B (Sect. 2) are displayed in the top and bottom panels, respectively. The global structure is consistent between both cases, as was expected from the similarities in the large-scale magnetic field distributions. However, a detailed comparison reveals 

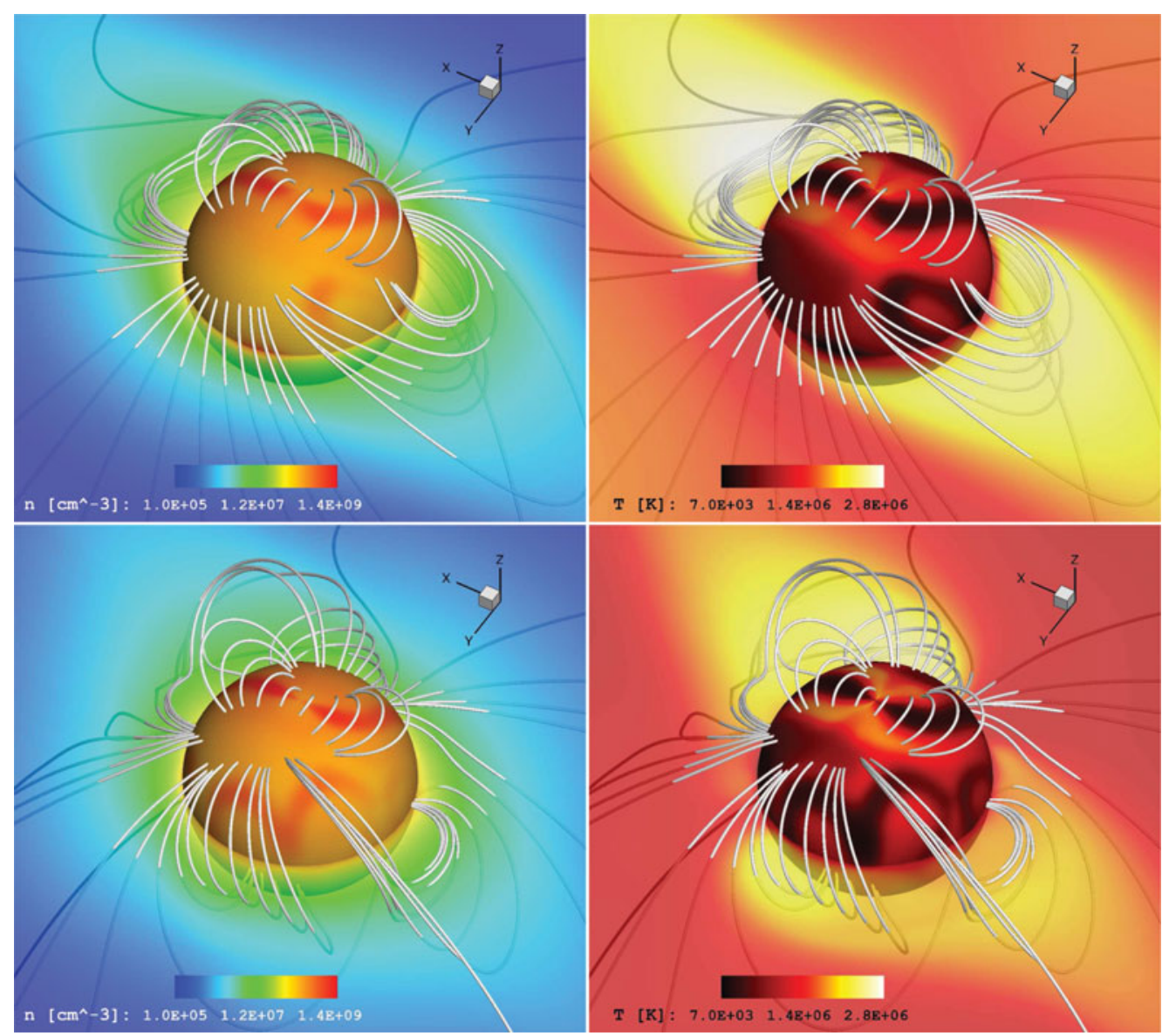

$T[K]: 7.08+03 \quad 1.4 E+06 \quad 2.8 E+06$

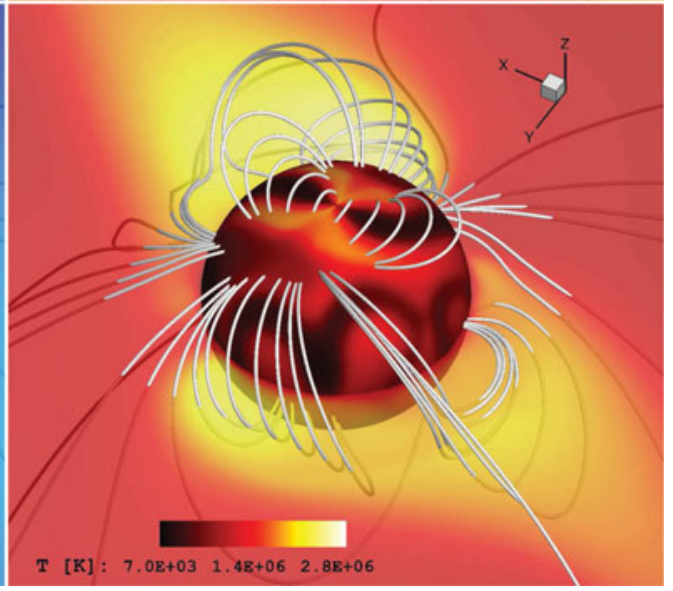

Figure 2. Results from the steady-state MHD solutions of the inner corona of GJ 3021 for cases A (top) and B (bottom). The distribution of the plasma density $(n)$ and temperature $(T)$, are shown in the left and right panels, respectively. The equatorial plane $(z=0)$ and the stellar surface are presented. Selected three-dimensional magnetic field lines are shown in white. See the on-line edition of this book for a color version of this figure.

significant differences in the final solutions. The coronal structure for case A (weaker field, less complexity, lower spatial resolution) contains a $\sim 20 \%$ hotter and $\sim 30 \%$ denser plasma in the inner region (Fig. 2), in comparison with case B (stronger field, increased complexity, higher spatial resolution). Cold and dense features are recovered near the main polarity inversion lines on the surface, resembling solar filaments or prominences. They result from plasma confined by the coronal magnetic field, as can be seen from the arcade loops near the north pole of the star. Due to the higher field complexity, these features are more common in case B. This surface complexity (and field strength) is reflected as well in the coronal field lines connectivity, leading to more open field lines in case A and larger and stretched loops in case B.

The stellar wind structure is shown in Fig. 3. The left panel contains the radial wind speed, $U_{r}$, projected onto the cartesian planes. As is indicated by the colour scale, the solution obtained for case A leads to a faster wind in comparison with case B $(\sim 20 \%$ difference). Therefore, due to mass conservation, the slow-wind region emerging from coronal streamers (dark-blue \& black in Fig. 3, left), is considerably larger in the second 


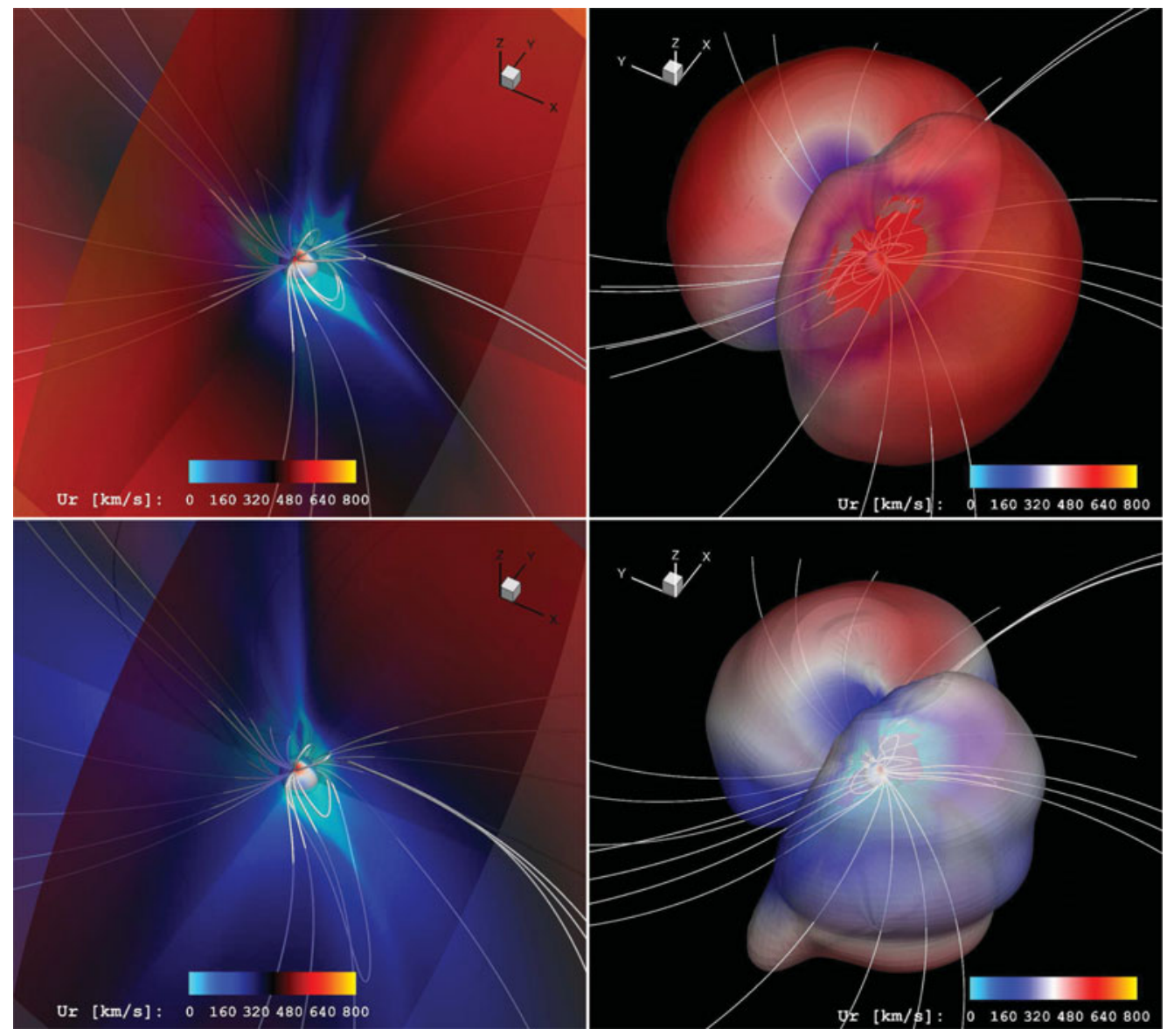

Figure 3. Results from the steady-state MHD solutions of the wind structure of GJ 3021 for cases A (top) and B (bottom). The sphere represents the stellar surface, coloured with the considered input ZDI maps (Fig. 1). We show the radial wind speed $\left(U_{r}\right)$ projection on the cartesian planes (left) and over the Alfvén surface (right). Selected three-dimensional magnetic field lines are shown in white. See the on-line edition of this book for a color version of this figure.

case. Moreover, given the magnetic field strength difference between both cases $(\sim 30 \%)$, larger coronal loops can be sustained in case B (Fig. 3, lower left panel).

The right panels of Fig. 3 contain the projection of $U_{r}$ over the Alfvén surface structure (where the Alfvénic Mach number, $M_{A}$, is equal to 1). The topology of the Alfvén surface is different in both cases. Larger and fairly symmetric lobes result in case A. Case B also shows two large lobes but is less symmetric and more complex. On average the wind speed, at the Alfvén surface, is lower by a factor of $3 \times$ in case B. This difference will have a strong impact on the dynamic pressure of the stellar wind $\left(\propto \rho U^{2}\right)$, and therefore in the size of the astrosphere of the system.

\section{Conclusions and Next Steps}

We have performed a numerical 3D MHD simulation of the corona and wind structure of the Sun-like exoplanet-host GJ 3021. The model has been driven by the surface magnetic field recovered with Zeeman Doppler Imaging (ZDI). We studied two cases by considering two different ZDI input maps (with similar large-scale structures but different spatial 
resolutions, complexity and field strengths). We find that the global structure of the inner corona is similar in both cases. However, the increased complexity, spatial resolution and strength of the surface field is translated to the coronal structure, leading to a larger fraction of closed field lines and trapped material near the surface. Similar results have been found in the case of the Sun, where the coronal structure is affected by the fine-scale surface magnetic structure (Garraffo et al. 2013). In addition, a double-lobed structure of the Alfvén surface is found in both cases, with clear differences in its complexity. The average wind speeds are a factor of $3 \times$ smaller in the model associated with the more complex, stronger magnetic field map. These differences can have also a strong impact in the mass loss and angular momentum loss rates due to the size of the lever arm that applies a torque on the star to spin it down (Cohen \& Drake 2014). An estimate of these quantities will be presented in future work, in addition to the dynamics of the astrosphere, outflows, and the conditions experienced by the exoplanets around this system.

\section{Acknowledgements}

The simulations have been carried out on the computing facilities of the Computational Center for Particle and Astrophysics (C2PAP) of the Excellence Cluster Universe (Germany).

\section{References}

Alvarado-Gómez, J. D., Grunhut, J., Hussain, G. A. J., et al. 2015, A\&A A (submitted)

Cohen, O., Drake, J. J., Kashyap, V. L., Hussain, G. A. J., C., \& Gombosi, T. I. 2010, ApJ 721, 80

Cohen, O. \& Drake, J. J. 2014, ApJ 783, 55

Cohen, O., Drake, J. J., Glocer, A., et al. 2014, ApJ 790, 57

Garraffo, C., Cohen, O., Drake, J. J., \& Downs, C. 2013, ApJ 764, 32

Ghezzi, L., Cunha, K., Smith, V. V., de Araújo, F. X., Schuler, S. C., \& de la Reza, R. 2010, ApJ 720,1290

Hussain, G. A. J., Jardine, M., Donati, J.-F., et al. 2007, MNRAS 377, 1488

Hussain, G. A. J., Collier Cameron, A., Jardine, M. M., et al. 2009, MNRAS 398, 189

Hussain, G. A. J. \& Alecian, E. 2014, in: M. Jardine, P. Petit \& H. Spruit (eds.), Magnetic Fields Throughout Stellar Evolution Proc. IAU Symposium No. 302 (France), p. 25

Jordan, C., Sim, S. A., McMurry, A. D., \& Aruvel, M. 2001, MNRAS 326, 303

Naef, D., Mayor, M., Pepe, F., Queloz, D., Santos, N. C., Udry, S., \& Burnet, M. 2001, A\&A 375,205

Powell, K. G., Roe, P. L., Linde, T. J., Gombosi, T. I., \& De Zeeuw, D. L. 1999, J. of Computational Phys. 154, 284

Shaviv, N. J. 2003, J. Geophys. Res 108, 1437

Sokolov, I. V., van der Holst, B., Oran, R., et al. 2013, ApJ 764, 23

Terada, N., Kulikov, Y. N., Lammer, H., Lichtenegger, H. I. M., Tanaka, T., Shinagawa, H., \& Zhang, T. 2009, Astrobiology 9, 55

Torres, C. A. O., Quast, G. R., da Silva, L., de La Reza, R., Melo, C. H. F., \& Sterzik, M. 2006, A $\& A$ A 490, 695

Tóth, G., van der Holst, B., Sokolov, I. V., et al. 2012, J. of Computational Phys. 231, 870

van der Holst, B., Sokolov, I. V., Meng, X., Jin, M., Manchester, IV, W. B., Tóth, G., \& Gombosi, T. I. $2014, A p J 782,81$

Vidotto, A. A., Jardine, M., Opher, M., Donati, J. F., \& Gombosi, T. I. 2011, MNRAS 412, 351

Vidotto, A. A., and Gregory, S. G., Jardine, M., et al. 2014, MNRAS 441, 2361

Wood, B. E., Müller, H.-R., Redfield, S., \& Edelman, E. 2014, ApJ 781, L33 